\title{
Study of resolution of the PANDA GEM detector with Garfield
}

\author{
D. Melnychuk $\cdot$ B.Voss $\cdot$ B. Zwieglinski
}

Published online: 6 May 2014

(C) The Author(s) 2014. This article is published with open access at Springerlink.com

\begin{abstract}
The forward GEM tracker of the PANDA detector at the future FAIR facility will track the particles produced in antiproton-proton annihilations and emitted in the polar angle range $5^{\circ}-22^{\circ}$. Position resolution at the level of $100 \mu \mathrm{m}$ and good time resolution are critical to work under luminosities up to $2 \times 10^{32} \mathrm{~cm}^{-2} \mathrm{~s}^{-1}$. The simulations performed with Garfield program compared several detector layouts and determined the optimal granularity of readout electronics. The time resolution for two possible gas mixtures was also estimated.
\end{abstract}

Keywords PANDA experiment · GEM detector · Garfield simulation

\section{Introduction}

The PANDA (AntiProton ANnihilation at DArmstadt) experiment [1] is one of the key projects at the future Facility for Antiproton and Ion Research, which is currently under construction at GSI, Darmstadt.

The $\bar{P}$ ANDA experiment will perform precise studies of antiproton-proton annihilations and reactions of antiprotons with nucleons of heavier nuclear targets with the centre-ofmass energy between $2.3 \mathrm{GeV}$ and $5.5 \mathrm{GeV}$. Particles emitted at angles covering $5^{\circ}-22^{\circ}$, which are not covered fully by the tracking detectors (STT and MVD), will be tracked by three planar GEM stations placed down-stream of the target. Each station consists of double

Proceedings of the 11th International Conference on Low Energy Antiproton Physics (LEAP 2013) held in Uppsala, Sweden, 10-15 June, 2013

D. Melnychuk $(\bowtie) \cdot$ B. Zwieglinski

NCBJ, Hoza 69, Warsaw, Poland

e-mail:dimam@fuw.edu.pl

B. Voss

GSI, Darmstadt, Germany 
planes with two projections per plane. The stations will be equipped with gaseous micropattern detectors based on Gas Electron Multiplier (GEM) foils as amplification stages. The chambers have to sustain a high counting rate of particles peaked at the most forward angles due to the relativistic boost of the reaction products as well as due to the small angle $\bar{p} p$ elastic scattering.

\section{GEM simulations with Garfield}

Creation and propagation of an electron avalanche and its collection by a readout plane in a GEM detector has been simulated with a Garfield program [2] to study its properties, namely space resolution and time performance. A standard design GEM has been assumed in simulations with a $50 \mu \mathrm{m}$ thick Kapton foil covered on both sides with $5 \mu \mathrm{m}$ copper layer. The holes are spaced so as to have a distance of $140 \mu \mathrm{m}$ between their centres. Each GEM hole has a double conical shape with an outer diameter of $70 \mu \mathrm{m}$ and an inner diameter of $50 \mu \mathrm{m}$. In the current design of the PANDA GEM detector the thickness of the drift volume is $1 \mathrm{~cm}$ and distances between GEM planes and between the last GEM plane and anode are $0.2 \mathrm{~cm}$. Simulations were performed with Garfield ++ with the electric field calculated with the ANSYS program [3].

Two gas mixtures were tested: $\mathrm{Ar} / \mathrm{CO}_{2}-70 \% / 30 \%$ and an alternative option with a $\mathrm{CF}_{4}$ admixture. The voltage setting on electrodes was chosen to create the field strength of $2.49 \mathrm{kV} / \mathrm{cm}$ in the drift volume and $3.73 \mathrm{kV} / \mathrm{cm}$ between the GEM foils and between the last GEM foil and the readout plane. No B-field was considered in the simulations. $1 \mathrm{GeV}$ muons directed perpendicular to the detector plane were assumed as primary tracks, which results on average in 29 primary electrons produced in the drift volume. This number results from a calculation with the HEED program [4], which is an implementation of the photoabsorption ionization (PAI) model. In the given simulation the transport of delta electrons was not included. Production of electrons by the primary muon track is neglected after the first GEM plane.

\section{Position resolution}

Production of a readout plane for the GEM detector with high granularity and the total size of the order of $1 \mathrm{~m}$ presents a technological challenge. Dependence of the detector resolution on the readout granularity was studied by increasing in steps the size of readout elements to note at which size it exceeded the goal of $100 \mu \mathrm{m}$. Position resolution is defined by the distribution of charge on the readout plane. It is determined by two main processes: a directed drift of electrons in the electric field and their random diffusion in the gas filling the detector. The diffusion of electrons in gas is followed microscopically for each produced electron, including diffusion of primary electrons in the drift volume, using transport parameters calculated with the Magboltz program [5], which interfaced into Garfield. As a result of simulations the 2D-gaussian distribution of charge on the readout plane is obtained with $\sigma=264 \mu \mathrm{m}$. 2-dimensional readout structure was studied with the strips oriented along the $0 Y$ axis and the pads connected along the $0 X$ axis. The primary muon position was uniformly distributed over the width of the electrodes.

The reconstructed track position is determined as a weighted mean of a cluster, i.e. the group of neighboring electrodes with a signal above the threshold. The level of threshold is determined by the noise of readout electronics, which is currently foreseen at the level of 
Table 1 Space resolution of the triple GEM detector for different pitch sizes of the readout

\begin{tabular}{lll}
\hline Pitch size, $\mu m$ & r.m.s.(x), $\mu m$ & r.m.s.(y), $\mu m$ \\
\hline 400 & 38 & 38 \\
600 & 40 & 41 \\
800 & 91 & 46 \\
1000 & 169 & 92 \\
\hline
\end{tabular}

$1000 e^{-}$. The gain of 10000 was assumed for the study of position resolution with variation of $10 \%$. To characterize the space resolution of different readout geometries the distribution of residuals between the generated and the reconstructed track position was built and the width of this distribution was taken to characterise the space resolution.

The results collected in Table 1 indicate that the pitch size of readout in the range 400$600 \mu \mathrm{m}$ satisfies the required space resolution. An irregular growth of resolution in $0 X$ and $0 Y$ for the larger pitch sizes is due to the decreasing number of electrodes contributing to the weighted mean position of the cluster, i.e. up to 5 electrodes can contribute to a hit for the smallest pad size, while all the signal happens to be collected on one electrode for the largest pad size. Introducing the definition of a valid hit, i.e. that the signal is above threshold at least in two pads, the reconstruction efficiency drops from $100 \%$ for 400 and $600 \mu \mathrm{m}$ pad widths to $84 \%$ for $800 \mu \mathrm{m}$ and down to $66 \%$ for the $1000 \mu \mathrm{m}$ pad width.

\section{Time performance}

Two questions should be studied related to the detector time performance: time resolution and high rate detector performance. The time performance is affected by several parameters of the detector: gas mixture; size of the drift volume, which defines the jitter of a signal; and the parameters of electronics, namely shaping time of the preamplifier.

A signal induced in the single readout strip of the width of $200 \mu \mathrm{m}$ was studied for two gas mixtures: $\mathrm{Ar} / \mathrm{CO}_{2}-70 \% / 30 \%$ and with an admixture of $\mathrm{CF}_{4}$, which is known to accelerate the collection, $\mathrm{Ar} / \mathrm{CO}_{2} / \mathrm{CF}_{4}-60 \% / 20 \% / 20 \%$. To study time resolution the following processing of the signal by readout electronics has been taken into account. The readout with a CR-RC shaper was assumed with an $18 \mathrm{~ns}$ shaping time and the noise level of $1000 e^{-}$. Time resolutions obtained for the two gas mixtures are 12.9 and $9.9 \mathrm{~ns}$ correspondingly. The average signal length for $\mathrm{Ar} / \mathrm{CO}_{2} / \mathrm{CF}_{4}$ gas mixture is also shorter than for $\mathrm{Ar} / \mathrm{CO}_{2}, 140$ vs $160 \mathrm{~ns}$.

\section{Conclusion}

The performed simulations estimated the optimal granularity of the readout electrodes for the GEM detector to be in the range 400-600 $\mu \mathrm{m}$, which is a compromise between the desired best resolution and technological difficulties to produce readout electrodes of high granularity. A comparison of the two different gas mixtures indicates advantages of $\mathrm{Ar} / \mathrm{CO}_{2} / \mathrm{CF}_{4}$ from the point of view of a better time resolution (9.9 ns) and also shorter signal length, which is an advantage in high rate measurements. 
Open Access This article is distributed under the terms of the Creative Commons Attribution License which permits any use, distribution, and reproduction in any medium, provided the original author(s) and the source are credited.

\section{References}

1. Lutz, M.F.M., et al., PANDA Collaboration: Physics Performance Report for PANDA: Strong Interaction Studies with Antiprotons. arXiv:0903.3905 [hep-ex]

2. Veenhof, R.: GARFIELD, recent developments. Nucl. Instr. Meth. A 419, 726-730 (1998)

3. Ansys: http://www.ansys.com/

4. Smirnov, I.B.: Modeling of ionization produced by fast charged particles in gases. Nucl. Instr. Meth. A 554, 474-493 (2005)

5. Biagi, S.F.: Monte Carlo simulation of electron drift and diffusion in counting gases under the influence of electric and magnetic fields. Nucl. Instr. Meth. A 421, 234-240 (1999) 